\title{
Effect of SiPM correlated noise and Photo-Detection Efficiency into charge resolution
}

\section{A. Nagai, ${ }^{a, *}$ C. Alispach, ${ }^{a}$ M. Dalchenko, ${ }^{a}$ D. della Volpe, ${ }^{a}$ M. Heller, ${ }^{a}$} L. D. M. Miranda, ${ }^{a}$ and T. Montaruli ${ }^{a}$

${ }^{a}$ Départment de physique nucléaire et corpusculaire, Université de Genève, 24 Quai E. Ansermet, CH-1211, Switzerland

E-mail: Andrii.Nagai@unige.ch

This paper reports on the studies of the effect of SiPM photon detection efficiency and correlated noise on the charge resolution of cameras for Imaging Atmospheric Cherenkov Telescopes (IACTs) with the goal of finding the optimal over-voltage (difference between applied and breakdown voltages) value providing the best balance between these two parameters. SiPM devices are more robust against light than photomultipliers, and allow to achieve longer exposure to gamma-ray sources more easily than photomultipliers (PMTs). Nonetheless, their sensitivity in the range of wavelength beyond $500 \mathrm{~nm}$, where the Night Sky Background increases, is higher than PMTs. Hence, filters on the camera entrance window can be used combined with anti-reflective and a dichroic coatings to cut out wavelengths longer than $\sim 540 \mathrm{~nm}$. The importance of such coatings and their effect on the charge resolution are also discussed in this article. This study is done with the Monte Carlo simulation (i.e. sim_telarray - simulation of the imaging atmospheric Cherenkov technique) and validated with measurements in the laboratory with calibrated light sources (one to mimic Cherenkov light and another for NSB). The studies are performed using SiPM devices produced by Hamamatsu and FBK. Results are compared with PMTs.

$37^{\text {th }}$ International Cosmic Ray Conference (ICRC 2021)

July 12 th - 23rd, 2021

Online - Berlin, Germany

\footnotetext{
${ }^{*}$ Presenter
} 


\section{Introduction}

Silicon Photomultipliers (SiPMs) have become the preferred photosensors for many applications in high-energy particle and astroparticle physics, LIDAR and medical imagining. Due to their robustness, low working voltage, ability to work with high continuous background light (i.e. during moon light) [7] and high photon detection efficiency, SiPM devices are good choice for cameras of Imaging Atmospheric Cherenkov Telescopes (IACTs) as pioneering works of FACT [1], SST-1M [5] and other small size telescope projects in CTA, such a ASTRI [3] and GATE-CHEC(GCT) [9], demonstrated. However, the over-voltage $\Delta V$ (difference between applied and breakdown voltages) affects almost all device parameters (more details in Ref. [8]) like gain, PDE, uncorrelated and correlated noise. In particular, by increasing the $\Delta V$ a the PDE can peak at about $60 \%$ (See Ref. [6]) can be reached. On the other hand, high $\Delta V$ leads to higher correlated noise, affecting image reconstruction.

In this work we study the optimal $\operatorname{SiPM} \Delta V$ value to provide the best balance between $P D E$ and the optical cross talk probability $P_{X T}$. We neglect the effect of SiPM uncorrelated noise or $D C R$ due to its minor values with respect to Night Sky Background or NSB. Also, we neglect SiPM afterpulse probability $P_{A P}$ (second source of correlated noise), because afterpulses are not contributing into image reconstruction due to low $P_{A P}$ and relatively long time constant $\tau_{A P}$ with respect to pulse falling edge (i.e. for Hamamatsu S10943-2832(X) the $P_{A P}$ of $8 \%$ at $P_{X T}$ of $25 \%$ was found in Ref. [8], with $\tau_{A P}=6.8 \mathrm{~ns}$ while for this study, the falling part of only $2 \mathrm{~ns}$ was used in integration window, after shaping of the signal). The study is done with a Monte Carlo simulation based on sim_telarray [2]. The simulation is validated with measurements in the laboratory with calibrated light sources. In general, SiPM devices are more sensitive to NSB with respect to PMTs due to higher $P D E$ above $500 \mathrm{~nm}$. To overcome such limitation, camera entrance window can be coated with anti-reflective and a dichroic coating to cut out wavelengths longer than $540 \mathrm{~nm}$. The importance of such window and effect of $N S B$ is studied and presented in this paper.

\section{Charge resolution calculation}

Both experimental and simulated data are analysed from the waveform level. For each waveform the baseline $B$ is subtracted. $B$ is calculated as the average over the first 20 amplitude values $W_{k}$ in a waveform. The number of detected photo electrons p.e. from light source is calculated as:

$$
N_{\text {p.e. }}=\frac{Q_{\text {signal }}}{Q_{\text {p.e. }}} \cdot\left(1-\frac{P_{x t}}{1-P_{x t}}\right) \text {, }
$$

where $P_{x t}$ is SiPM optical crosstalk probability, $Q_{\text {signal }}$ is the integrated charge within time window were events are expected (i.e. flasher, Cherenkov, etc.) and $Q_{p . e .}$ single photo-electron p.e. charge. $N_{p . e}$. is converted into number of emitted photons $N_{\gamma}=\frac{N_{p . e .}}{\epsilon_{C h}}$, where $\epsilon_{C h}$. is Cherenkov cumulative efficiency. The $\epsilon_{C h}$. as well as $N S B$ cumulative efficiency $\epsilon_{N S B}$ are calculated in wavelength range from $300 \mathrm{~nm}$ up to $1000 \mathrm{~nm}$. It includes $P D E$ and filter transmissivity as a function of wavelength, fill-factor of $91.94 \%$, funnel transmissivity of $88.55 \%$ and spectrum weighted average reflectivity of $92.94 \%$. The charge resolution $Q R$ is defined as the ratio between standard deviation $\sigma_{p . e .}$ and 
expected average value of the reconstructed number of detected photons $\left\langle N_{\text {p.e. }}\right\rangle$, as:

$$
Q R=\frac{\sigma_{\text {p.e. }}}{\left\langle N_{\text {p.e. }}\right\rangle}=\frac{\sqrt{\frac{\sum_{n=0}^{N}\left(N_{i}-\left\langle N_{\text {p.e. }}\right\rangle\right)^{2}}{N_{\text {events }}}}}{\left\langle N_{\text {p.e. }}\right\rangle},
$$

\section{SiPM devices}

For the time being, four SiPM devices have been considered as the most promising for the IACTs:

- Hamamatsu devices with $50 \times 50 \mu \mathrm{m}^{2}$ micro-cell sizes:

- LCT2 (Low cross-talk, second generation), hexagonal devices conceived for the SST$1 \mathrm{M}$ camera;

- LCT5 (Low cross-talk, fifth generation) device, with higher PDE than LSCT2 and low cross-talk;

- LVR3 (Low reverse voltage) devices, with the highest PDE at a given micro-cell size, but with high micro-cell capacitance and, hence, resulting long pulse shape, causing higher read-out electronics noise;

- FBK $N U V-H D$ device with $40 \times 40 \mu \mathrm{m}^{2}$ micro-cell size;

The peak $P D E^{1}$ as a function of $P_{X T}$ for all four devices is presented in Fig. 1 (left). Since, the $L C T 2$ device has the smallest $P D E$ and $L V R 3$ device has very long pulse length, we considered two devices $L C T 5$ and $N U V-H D$ as the most promising for this studies. Therefore, all further studies are presented only for these two devices. The optical efficiency $P D E \times \eta_{\text {window }}$ (where $\eta_{\text {window }}$ is camera entrance window transmissivity) for SiPM devices and PMT with or w/ and without or w/o filters at room temperature $\left(\mathrm{T}=25^{\circ} \mathrm{C}\right)$ as a function of wavelength $\lambda$ are presented in Fig. 1 (right).

\section{The Monte Carlo studies}

For the Monte Carlo studies the sim_telarray software is used. The studies are done with Light Emission package. In this studies, instead of Cherenkov showers, the flasher light is simulated. The flasher is located $5 \mathrm{~m}$ away from the camera to provide uniform illumination. Since, different SiPM devices have different $P D E$ vs. wavelength dependence (see Fig. 1(right)), the flasher light wavelength is set equal to Cherenkov spectrum. Simulations are done for various light intensities from few photons up to thousand of photons.

In the simulation, we implement a SiPM-camera, currently studied in the LST project [4], which features 7987 pixels, each of a linear size of $2.32 \mathrm{~cm}$ instrumented with an hexagonal SiPM. This shape ensures a spatial uniform trigger response of the camera. The large pixel size is achieved

\footnotetext{
${ }^{1}$ for Hamamatsu SiPM devices the peak $P D E$ was found at $\lambda=480 \mathrm{~nm}$, while for FBK device the peak PDE was found at $\lambda=400 \mathrm{~nm}$
} 

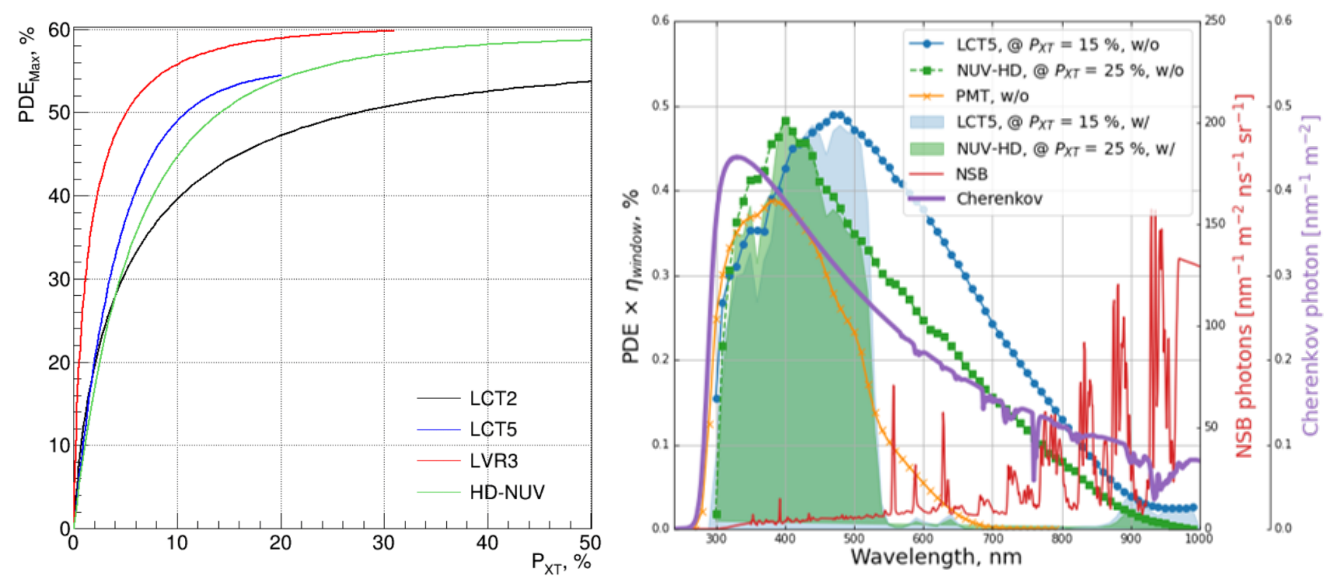

Figure 1: Left: Peak $P D E$ as a function of $P_{X T}$ for three hamamatsu devices: $L C T 2, L C T 5, L V R 3$ and FBK $N U V-H D$ device. The peak $P D E$ at $\lambda=480 \mathrm{~nm}$ was found for Hamamatsu SiPM devices, while for FBK device the peak $P D E$ was found at $\lambda=400 \mathrm{~nm}$. Right: The optical efficiency $P D E \times \eta_{\text {window }}$ (where $\eta_{\text {window }}$ is camera entrance window transmissivity) as a function of wavelength $\lambda$ for $L C T 5$ and NUV $-H D$ devices and PMT with or w/ and without or w/o filters. The Cherenkov and the spiky NSB spectrum typical of La Palma, Canarian islands, are shown as well in violet and red, respectively.

through a large SiPM of $93.6 \mathrm{~mm}^{2}$, coupled with a light funnel. Since, the SiPM devices are sensitive to red light, where the NSB is dominant, two configurations of the SiPM camera are used: with $(w /)$ and without $(w / o)$ filter on a camera entrance window. The filter cuts off wavelengths above $540 \mathrm{~nm}$. It was realized for the SSI-1M camera [5].

The simulations are provided for $P_{X T}$ of 5, 8, 15 and $25 \%$ and corresponding values of $P D E$ (see Fig. 1) for $L C T 5$ and NUV - HD devices. For each condition the NSB is calculated with and without filter. The obtained results are compared with the PMTs used in the LST camera, which adopts a PMMA (Shinkolite from Mitsubishi) window without filter. The summary of the simulated parameters is presented in Tab. 1. The cumulative efficiency is calculated as described in Sec. 2.

\section{Validation of the Monte Carlo}

\subsection{Experimental set-up}

For this study, the experimental setup is built and calibrated at the University of Geneva. The full description of the set-up may be found in [8]. The SiPM is biased with a Keithley 2410 through an RC filter $\left(R_{\text {bias }}=10 \mathrm{k} \Omega, C_{\text {bias }}=100 \mathrm{nF}\right)$. The SiPM anode is connected to the Keithley Picoammeter 6487 to measure $V_{\text {bias }}$ and also to the preamplifier board developed by INFN as one of the possible read-out solutions for the future LST SiPM camera. The waveform readout is performed with a Lecroy 620Zi oscilloscope. The set-up is equipped with a LED $(\lambda=630 \mathrm{~nm})$ and pico-second laser $(\lambda=375 \mathrm{~nm})$. A laser is in pulsed mode (to emulate the flashes of Cherenkov light induced by atmospheric showers), while the LED is in continuous, or in the so-called $D C$ mode, to emulate the $N S B$. The SiPM under test is operated at $V_{\text {bias }}=60.6 \mathrm{~V}$ and temperature of $25^{\circ} \mathrm{C}$, corresponding to $\Delta V=5.8 \mathrm{~V}\left(P_{X T}=25 \%\right)$. For each light level, 10 ' 000 waveforms are 


\begin{tabular}{|c|c|c|c|c|c|c|c|}
\hline \multirow{2}{*}{ Photo detector } & \multirow{2}{*}{ Window } & \multirow{2}{*}{\multicolumn{2}{|c|}{ Parameter }} & \multicolumn{4}{|c|}{$P_{X T}$} \\
\hline & & & & $5[\%]$ & $8[\%]$ & $15[\%]$ & $25[\%]$ \\
\hline \multirow{7}{*}{$L C T 5$} & \multicolumn{3}{|c|}{$P D E_{\text {Max }}[\%]$} & 35 & 47 & 53 & 54 \\
\hline & \multirow{3}{*}{$\begin{array}{l}\text { without } \\
\text { filter }\end{array}$} & \multicolumn{2}{|c|}{$\begin{array}{c}\text { NSB } \\
{[\mathrm{MHz} / \text { pixel] }}\end{array}$} & 261.33 & 386 & 424 & 437.7 \\
\hline & & \multirow{2}{*}{$\begin{array}{c}\text { cum. } \\
\text { efficiency }\end{array}$} & NSB [\%] & 4.83 & 6.83 & 8.57 & 8.85 \\
\hline & & & Cherenkov [\%] & 16.87 & 21.61 & 24.98 & 25.76 \\
\hline & \multirow{3}{*}{ with filter } & \multicolumn{2}{|c|}{$\begin{array}{c}\mathrm{NSB} \\
{[\mathrm{MHz} / \text { pixel }]}\end{array}$} & 73.4 & 107.6 & 109 & 110.3 \\
\hline & & \multirow{2}{*}{$\begin{array}{c}\text { cum. } \\
\text { efficiency }\end{array}$} & NSB [\%] & 1.35 & 2.1 & 2.69 & 2.76 \\
\hline & & & Cherenkov [\%] & 11.24 & 14.15 & 16.1 & 16.53 \\
\hline \multirow{7}{*}{$N U V-H D$} & \multicolumn{3}{|c|}{$P D E_{\text {Max }}[\%]$} & & 40 & 49 & 52 \\
\hline & \multirow{3}{*}{$\begin{array}{l}\text { without } \\
\text { filter }\end{array}$} & \multicolumn{2}{|c|}{$\begin{array}{c}\text { NSB } \\
{[\mathrm{MHz} / \text { pixel] }}\end{array}$} & & 160.4 & 224 & 271.4 \\
\hline & & \multirow{2}{*}{$\begin{array}{c}\text { cum. } \\
\text { efficiency }\end{array}$} & NSB [\%] & & 3.2 & 4.55 & 5.53 \\
\hline & & & Cherenkov [\%] & & 14.7 & 18.99 & 21.64 \\
\hline & \multirow{3}{*}{ with filter } & \multicolumn{2}{|c|}{$\begin{array}{c}\text { NSB } \\
{[\mathrm{MHz} / \text { pixel }]}\end{array}$} & & 65.1 & 83.8 & 94.7 \\
\hline & & \multirow{2}{*}{$\begin{array}{l}\text { cum. } \\
\text { efficiency }\end{array}$} & NSB [\%] & & 1.17 & 1.57 & 1.8 \\
\hline & & & Cherenkov [\%] & & 11.03 & 13.9 & 15.46 \\
\hline \multirow{4}{*}{$P M T$} & \multicolumn{3}{|c|}{$P D E_{\text {Max }}[\%]$} & \multicolumn{4}{|c|}{43} \\
\hline & \multirow{3}{*}{$\begin{array}{l}\text { without } \\
\text { filter }\end{array}$} & \multicolumn{2}{|c|}{$\begin{array}{c}\text { NSB } \\
{[\mathrm{MHz} / \text { pixel] }}\end{array}$} & \multicolumn{4}{|c|}{245.8} \\
\hline & & \multirow{2}{*}{$\begin{array}{c}\text { cum. } \\
\text { efficiency }\end{array}$} & NSB [\%] & \multicolumn{4}{|c|}{1.61} \\
\hline & & & Cherenkov [\%] & & & 67 & \\
\hline
\end{tabular}

Table 1: Summary of simulated sim_telarray parameters for two SiPM devices at different $P_{X T}$ values of 5,8,15 and $25 \%$ and PMT for two configurations: with and without filter. At $P_{X T}=5 \%$ only the $L C T 5$ device is studied. The cumulative efficiency is calculated in the wavelength range from $300 \mathrm{~nm}$ up to $1000 \mathrm{~nm}$. It includes $P D E$ and filter transmissivity as a function of wavelength, fill-factor of $91.94 \%$, funnel transmissivity of $88.55 \%$ and spectrum weighted average reflectivity of the mirrors of $92.94 \%$.

acquired, each of $100 \mathrm{~ns}$ long (1'000 samples per waveform). The light intensity is monitored with $5 \%$ precision using a calibrated photodiode ${ }^{2}$.

\subsection{Validation with calibrated light source}

For Monte Carlo validation, the experimental set-up described above is used. The $D C$ LED of $\lambda=630 \mathrm{~nm}$ is used to mimic the $N S B$, while a $375 \mathrm{~nm}$ laser mimics the Cherenkov signal. Since, devices with hexagonal shape and active area of $93.6 \mathrm{~mm}^{2}$ are only available in $L C T 2$, the validation is done for them at $P_{X T}$ of $25 \%$. The results are presented in Fig. 2. We can observe a good agreement between measured and simulated data.

${ }^{2}$ Hamamatsu S1337-1010BQ, s/n 61 


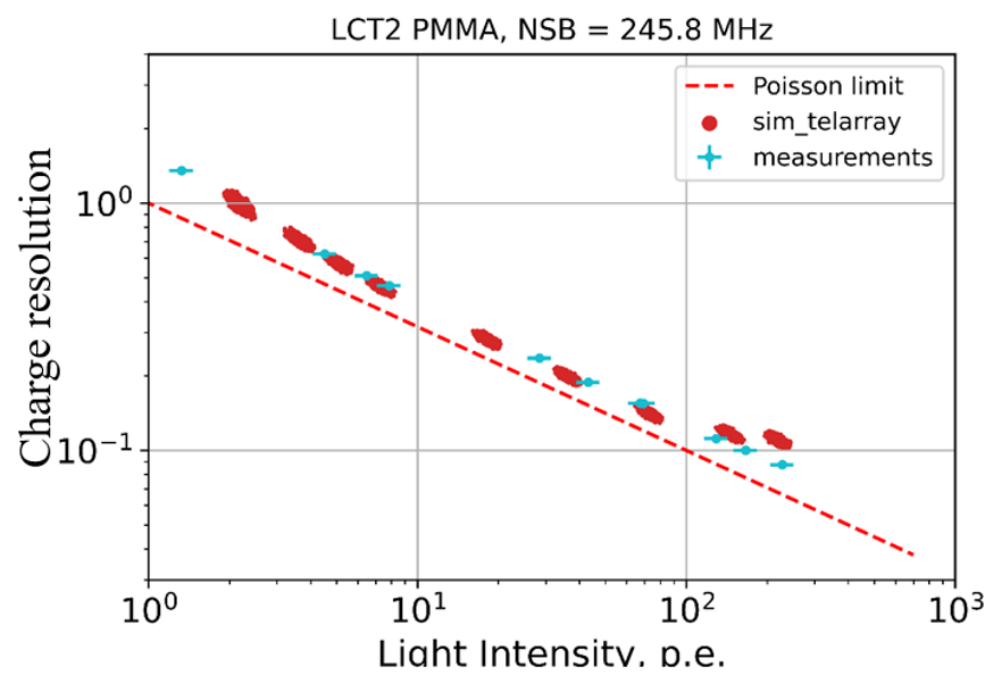

Figure 2: Comparison between simulated and measured $Q R$ as a function of detected number p.e. for $L C T 2$ device at $P_{X T}$ of $25 \%$.

\section{Results: Optimal $V_{\text {bias }}$ in terms of $P D E$ and $P_{X T}$}

Independently of the SiPM device and the value of $P_{X T}$, the best charge resolution $Q R$ as a function of detected photo-electrons p.e. is achieved with filter due to the reduction of the NSB contribution. Since, PMTs and SiPM devices have different active area, the $Q R$ was normalized into Light Guide area or $A_{\text {L.G. }}$, which is $4.67 \mathrm{~cm}^{2}$ for SiPM camera and $16.24 \mathrm{~cm}^{2}$ for PMT camera. As an example, the $Q R \times \sqrt{A_{L . G .}}$ as a function of p.e. $/ \mathrm{mm}^{2}$ for $P_{X T}=15 \%$ is presented in Fig. 3 (left) and compared with the Poisson limit $Q R_{P}$ (red dashed line), calculated as $Q R_{P}=\frac{1}{\sqrt{N_{p . e .}}}$, where $N_{p . e .}$ is the number of detected p.e.. Since, the simulations are performed for the full camera, at each light level 7987 data points are presented for the SiPM camera and 1855 data points for the PMT camera. We can observe, the deviation of $Q R$ from $Q R_{P}$ at very low (bellow 1 p.e. $/ \mathrm{mm}^{2}$ ) light intensity for configurations without filter due to the high cumulative $N S B$ efficiency. Nevertheless, the configurations without filter shows much better $Q R$ at high light intensity (after 10 p.e. $/ \mathrm{mm}^{2}$ ) due to the higher cumulative Cherenkov efficiency, leading to higher photon statistics. From such a representation it is very difficult to compare the configurations with different optical efficiency. Therefore, the $Q R$ should be presented as function of photons $\gamma$.

Independently of the value of $P_{X T}$, the lowest $Q R$ as a function of $\gamma$ is achieved for the "without filter' configuration with the $L C T 5$ device, due to the highest Cherenkov cumulative efficiency (See Tab. 1). Also, from those results, we can observe that high $N S B$ cumulative efficiency is not a problem, since all configurations show the same $Q R$ at low light intensity. As a matter of fact, in this region, the main source of $Q R$ degradation is the read-out electronics noise, while at high light intensity the $N S B$ rate is negligible compared to the Cherenkov signal. As an example the $Q R \times \sqrt{A_{L . G}}$. as a function of $\gamma / \mathrm{mm}^{2}$ for $P_{X T}=15 \%$ is presented in the Fig. 3 (right). We can observe that the $Q R \times \sqrt{A_{L . G}}$ for PMT based camera is in the same level as SiPM cameras with 
filters due to relatively close values of cumulative efficiency (both Cherenkov and $N S B$ ) and $N S B$ level per $\mathrm{mm}^{2}$.
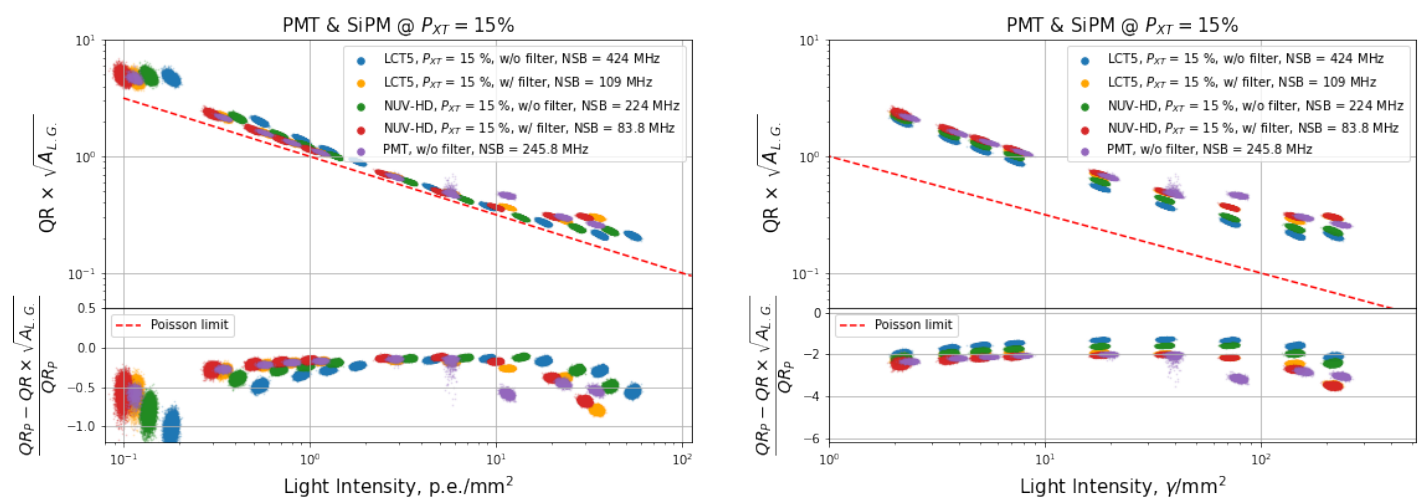

Figure 3: Charge resolution as a function of Cherenkov light intensity expressed in p.e. (left) and photons (right) for cameras with and without filters based on PMT and SiPM devices: LCT5 and NUV - HD. The $C T A$ requirements for LST telescope for dark night and half Moon nights are presented. The normalized difference, between obtained $Q R$ and Poisson limit $Q R_{P}$ are presented in bottom plot.

The $Q R$ as a function $\gamma$ for configurations which fulfill the CTA requirements is presented in Fig. 4. We can observe that the camera with the LCT5 devices meets the requirements at values of $P_{X T}$ of 8,15 and $25 \%$, while the camera based on $N U V-H D$ (which has PDE vs. $\lambda$ shape more suitable for Cherenkov signals, see Fig. 1(right)) satisfies the requirements only at $P_{X T}$ of 15 and 25\%. Also, independent of the SiPM device, the optimal $Q R$ is obtained for $P_{X T}$ of $15 \%$,

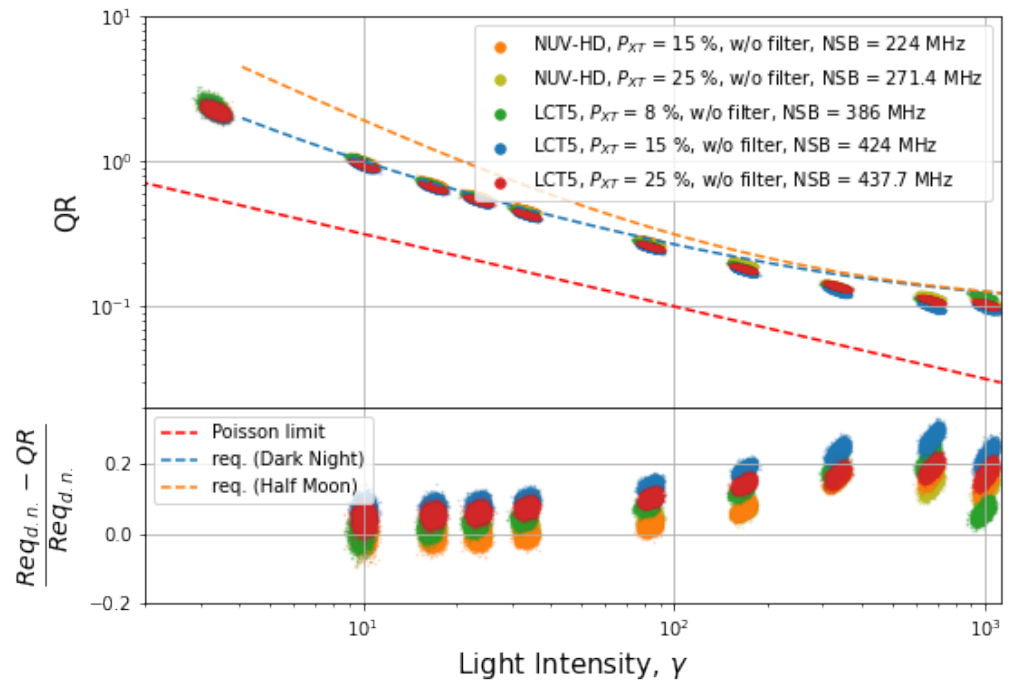

Figure 4: $Q R$ for configurations which full-fill the CTA requirements as a function of Cherenkov light intensity expressed in photons $(\gamma)$. 
because at this value of $P_{X T}$ the $P D E$ as a function of $P_{X T}$ starts to saturate (see Fig. 1 (left)). The configuration with $P_{X T}=15 \%$, shows $9 \%$ improvement in $Q R$ with respect to $P_{X T}=8 \%$ configuration, which was considered as the optimal one previously [5].

\section{Conclusions}

In this paper we report on the studies of the effect of SiPM $P_{X T}$ and $P D E$ on the charge resolution $Q R$ of Imaging Atmospheric Cherenkov Telescopes (IACTs). We found, that the optimal operational voltage of SiPM devices for IACTs is when $P D E$ vs. $P_{X T}$ starts to saturate. In particular, for studied $L C T 5$ and $N U V-H D$ devices it is at $P_{X T}=15 \%$. Also, we found that even if SiPM devices in general are more sensitive to $N S B$ with respect to PMTs, the filter is not needed. Moreover, the usage of the filter degrade the $Q R$ due to significant decrease of cumulative Cherenkov efficiency. Since, the $N S B$ affects $Q R$ only at low light intensity, while for all other light intensity the high cumulative Cherenkov efficiency is more preferable and leads into better $Q R$. Assuming that future IACTs will have the same optical properties as the one listed in Sec. 2, the LCT5 devices full-fill the CTA requirements at $P_{X T} \geqslant 8 \%$, while the $N U V-H D$ device at $P_{X T} \geqslant 15 \%$.

\section{References}

[1] Anderhub H., et al., 2013, JINST, 8, P06008

[2] Bernlhöhr K., 2008, Astrop. Phys., 30, 149

[3] Billotta S., et al., 2014, in Holland A. D., Beletic J., eds, Vol. 9154, High Energy, Optical, and Infrared Detectors for Astronomy VI. SPIE, pp 572 - 585, https://doi .org/10.1117/12. 2055331

[4] Heller M., et al., 2021, in in these proceedings, https://pos.sissa.it/395.

[5] M. Heller et al. 2017, The European Physical Journal C, 77, 47

[6] Nagai A., et al., 2018, Nuclear Instruments and Methods in Physics Research Section A: Accelerators, Spectrometers, Detectors and Associated Equipment, 912, 182

[7] Nagai A., Alispach C., Della Volpe D., Heller M., Montaruli T., Njoh S., Troyano-Pujadas I., Renier Y., 2019a, JINST, 14, P12016

[8] Nagai A., et al., 2019b, Nucl. Instr. Meth. A, 948, 162796

[9] Watson J., Zorn J., 2019, arXiv: Instrumentation and Methods for Astrophysics 